\title{
Hybrid Beamforming with G2+1+1 Transmission Scheme and Modified SIC Design for Milimeter Wave Channel
}

\author{
Francisco M. C. Caldas, Daniel C. Araujo and Walter C. Freitas Jr.
}

\begin{abstract}
The use of massive multiple input multiple output (MIMO) has been considered as a key enabler to achieve the 5G requirements [1]. With the large array of antennas, the beamformers' uses have become very attractive, specially when the energy is concentrated in a specific direction. However, implementing large arrays becomes prohibitive since the digitalanalog converters (DAC) are power hungry devices connected to every radio frequency (RF) chain. So to deal with this bottleneck, the number of RF chains is reduced by using the hybrid beamforming (HB) solution whose implementation enables us to use fewer RF chains than the digital solution while maintaining a high number of antennas. In this work, we propose to extend a diversity-multiplexing transmission scheme called as G2+1+1 for massive MIMO systems in millimeter wave (mmWave) channel. To implement such scheme, we design a Hybrid Beamforming at the base station and a nonlinear decoder at the receiver.

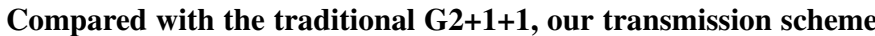
combines diversity, multiplexing and array gains into one unique device. We show in our simulations that our scheme outperforms in terms of bit-error rate (BER) other solutions that only exploit massive MIMO multiplexing gain. Such results show that the proposed solution is very attractive to increase the system reliability and provides enhanced robustness in low and high SNR scenarios.
\end{abstract}

Index Terms-Hybrid Beamforming, Modified SIC, G2+1+1, Millimeter Wave.

\section{INTRODUCTION}

$\mathbf{T}$ HE data rate consumption in mobile communication systems is growing exponentially, and fifth generation (5G) systems need to provide technical solutions to follow this growth. The mmWave communication solutions has the potential to offer gigabit-per-second data rates by exploiting the large bandwidth available at mmWave frequencies. However, the mmWave channel communication at high frequencies suffers from high attenuation and signal absorption and it has power-limited due to path loss associated with the wavelengths of the channel [2]. To compensate the significant path loss, very large antenna arrays can be used at the base station (BS) and the mobile station (MS) to exploit beam steering and increase the array gain. The use of large arrays, also called massive multiple input multiple output (MIMO), has been considered as a key enabler to achieve the $5 \mathrm{G}$ requirements

Francisco M. C. Caldas is supported by CAPES (Brazil). The authors are with the Department of Teleinformatic and the Group of Wireless Communication Research (GTEL) at the Federal University of Ceará, Fortaleza, Brazil. E-mails: \{fcomarcio,araujo,walter\}@gtel.ufc.br

The Associate Editor coordinating the review of this manuscript and approving it for publication was Prof. José Cândido Santos Filho.

Digital Object Identifier: 10.14209/jcis.2017.8
[1]. The gains provided by the MIMO systems are well known but due to variability of the channel states, there is a need of some coordination to choose the most indicated gain at a certain time. However, when the number of antennas increase extremely, those gains are obtained with almost any coordination [3]. In the same way, one other possibility to avoid coordination is consider transmission schemes that combines diversity and multiplexing in the same transceiver.

In classical MIMO, these techniques of combining diversity and multiplexing have been used to improve the system reliability keeping at the same time a high data rate. For instance, we can mention the scheme that transmits two multiantenna layers using spatial multiplexing i.e., vertical bell-labs layered space-time (VBLAST) [4], [5] and one multi-antenna layer using space-time block code (STBC) i.e., Alamouti coding [6]. Therefore, we can get jointly the diversity of the STBC and the data rate of the VBLAST scheme. However, the classical implementation for this scheme consists of using a four transmit antenna MIMO system, which is a number extremely low for the massive MIMO case.

With the large array of antennas, the beamformers' uses have become very attractive, specially when the energy is concentrated in a specific direction. However, the use of digital beamforming requires one radio frequency (RF) chain per antenna which means an inefficient solution in terms of energy consumption, for the number of digital-analog converters (DAC)s circuits used. The converters are power hungry devices connected to every RF chain, so their power consumption in implementing large arrays becomes prohibitive. To deal with this bottleneck, the number of RF chains is reduced by using some hybrid beamforming (HB) solution composing analog and digital filters whose implementation enables us to user fewer RF chains than the full digital solution while maintaining a high number of antennas. Although, this scheme imposes hardware limitations that may cause reduction of the system achievable rate, there are works showing achievable rates similar to those obtained by using a pure digital beamforming solution [2], [7]. Some works on HB propose new solutions either interested on achieving the maximum spatial multiplexing gain or the maximum diversity gain [8]. For instance, the authors in [9] consider the VBLAST solution to achieve the multiplexing gain while other works, such as in [10]-[12], present HB solutions based on the STBC scheme to obtain the diversity gain.

In this work, we propose a multi-layer beam transmission scheme for massive MIMO systems that exploits, at the same 
time, spatial multiplexing and diversity gains. The transmission is done by means of hybrid beamforming solution, so the large array gain can be used to steer the signal into a specific direction. The signal adopted in this paper follows the $\mathrm{G} 2+1+1$ [6] configuration that provides at the same time spatial multiplexing and diversity gains. Therefore, the proposed solution exploits jointly the beamforming, spatial, and diversity gains. The receiver employs a decoder that implements a nonlinear algorithm called backward recursion successive interference cancellation (BRSIC), which was proposed in [13]. This algorithm is an enhanced version of the well known successive interference cancellation (SIC) which is used as a VBLAST detection solution as shown in [14]-[16]. Basically, the BRSIC has an additional stage, compared to the traditional SIC, to remove the remaining inter-layer interference providing fulldiversity at the receiver to all multi-layers. The results obtained in our work, show a system reliability improvement specially in low signal-to-noise ratio (SNR) and low angular spread scenarios, such as millimeter wave channels [17]. Another advantage of this method is the design of the HB which is based on the partial knowledge of the channel. In such a case, the base station (BS) does not need to estimate the full massive MIMO channel which reduces the number of pilot sequences needed to estimate the channel [18].

The remaining of the work is organized as follows. In Section II, we describe the system model, present the HB structure, and the channel model used. In Section III, we explain the nonlinear detection named modified backward recursion successive interference cancellation (BRSIC). In Section IV, we present the simulation results of the proposed hybrid beamforming transmission solution in high and low angular spread scenarios. Finally, Section V finalizes this paper with conclusions and perspectives.

\section{SySTEM MODEL}

Consider a BS with $N_{B S}$ antennas and a MS with $N_{M S}$ antennas. Assume that the number of antennas at the BS is much larger than that in the MS, i.e., $N_{B S} \gg N_{M S}$. The MS implements a full digital beamforming and the BS employs a HB with $M$ RF chains. The BS uses a multi-layer beam transmission scheme to serve the MS while the MS decodes the signal using a full digital beamforming solution. The multibeam layer scheme is composed by $J=4$ layers, where $K$ data streams that are split into layers. The number of layers is such that $K \leq M \leq N_{B S}$ and $K \leq N_{M S}$ [19]. The BS transmits the set of streams using a $\mathrm{G} 2+1+1$ scheme as shown in Fig. 1. This scheme encodes a given symbol vector $\mathbf{S}=\left[s_{1}, s_{2}, s_{3}, s_{4}, s_{5}, s_{6}\right]$ and generates a space-time codeword matrix as

$$
\mathbf{X}(\mathbf{s})=\left[\begin{array}{cccc}
s_{1} & s_{2} & s_{3} & s_{4} \\
-s_{2}^{*} & s_{1}^{*} & s_{5} & s_{6}
\end{array}\right]^{T}
$$

Note that, the $\mathrm{G} 2+1+1$ encoder uses two RF chains to transmit two data stream over an Alamouti's STBC scheme and two others RF chains to transmit two data streams using spatial multiplexing transmission scheme [20].

The covariance matrix of the symbols is $E\left[\mathbf{s s}^{H}\right]=\left(\frac{P_{S}}{K}\right) \mathbf{I}_{K}$, where $P_{S}$ is the total transmit power. The codeword matrix is

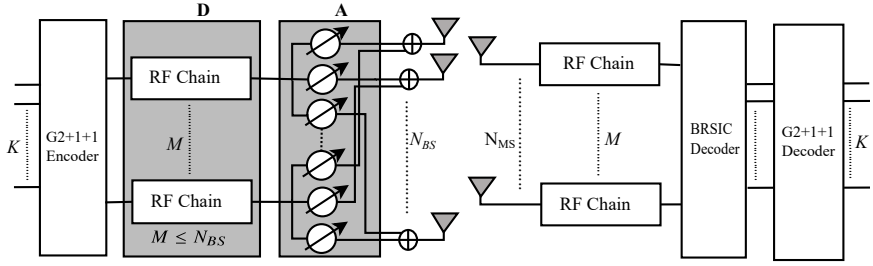

Figure 1. Simplified block diagram of the system with the BS employing $\mathrm{G} 2+1+1$ scheme followed by hybrid beamforming and MS using a BRSIC decoder followed by $\mathrm{G} 2+1+1$ decoder scheme.

the input of the transmitter beamforming $\mathbf{F}=\mathbf{A D}$, where $\mathbf{A}$ is the analog beamforming and $\mathbf{D}$ is the digital beamforming. The matrix $\mathbf{A} \in \mathbb{C}^{N_{B S} \times M}$ is implemented using a phaseshifter network that connects every RF chain to every antenna element. In this part, only the signal phase is possible to adjust, thus all the entries in $\mathbf{A}$ are constant modulus and consequently no amplitude adjustment can be performed. Whereas for the digital part, the matrix $\mathbf{D} \in \mathbb{C}^{M \times 4}$ is implemented using a set of DSPs which does not impose modulus restrictions on entries of D. Finally, the received signal at the MS is denoted for

$$
\mathbf{Y}=\mathbf{H A D X}(\mathbf{s})+\mathbf{Z}
$$

where $\mathbf{Z} \in \mathbb{C}^{N_{M S} \times K}$ is the corrupting Gaussian noise at the received signal and $\mathbf{H} \in \mathbb{C}^{N_{M S} \times N_{B S}}$ is the channel matrix that can be assumed as a channel to millimeter wave frequencies [2].

We consider a scenario with $L$ scatterers in which each one has a single propagation path that connects the BS and MS [21].We assume a flat-fading channel model for the ease of the problem exposition; however, the proposed technique can be extended for the frequency selective case by assuming an orthogonal frequency division multiplexing (OFDM). The channel model is expressed as

$$
\mathbf{H}=\sqrt{\frac{N_{B S} N_{M S}}{\rho}} \sum_{l=1}^{L} \alpha_{l} \mathbf{a}_{M S}\left(\theta_{R X, l}\right) \mathbf{a}_{B S}^{H}\left(\theta_{T X, l}\right),
$$

where $\rho$ is the average path-loss between the BS and MS, $\alpha_{l} \sim \mathcal{N}\left(0, P_{R}\right)$ is the complex gain of the $l^{\text {th }}$ path with $l=1,2, \ldots, L$ and $P_{R}$ your variance. The angles of departure and arrival (AoDs/AoAs) are $\theta_{T X, l} \in[0,2 \pi]$ and $\theta_{R X, l} \in[0,2 \pi]$, respectively. The steering vectors $\mathbf{a}_{B S}\left(\theta_{T X, l}\right)$ or $\mathbf{a}_{M S}\left(\theta_{R X, l}\right)$ represent an uniform linear arrays (ULA) and can be represented as

$$
\begin{aligned}
\mathbf{a}_{B S}\left(\theta_{T X, l}\right)= & \frac{1}{\sqrt{N_{B S}}}\left[1, e^{j(2 \pi / \lambda) d \sin \left(\theta_{T X, l}\right)}, \ldots\right. \\
& \left.\ldots, e^{j\left(N_{B S}-1\right)(2 \pi / \lambda) d \sin \left(\theta_{T X, l}\right)}\right]^{T},
\end{aligned}
$$

where $\lambda$ is the signal wavelength and $d$ is the distance between antenna elements.

The design of the analog beamforming $\mathbf{A}$ is based uniquely on the angular information of the transmitter side, $\theta_{T X, l}$. Although the estimation of such information plays an important role for designing the transmitter, we assume perfect knowledge of the transmitted angles at the BS. It chooses a set of paths by estimating the $M$ strongest ones [22] and 
determines the phase-shifter values according to the steering vector of the chosen directions. This approach is attractive because the steering vector naturally meets the constant modulus restriction since the amplitude of each element is equal as shown in Eq. (4). Moreover, the BS does not require knowledge of the full massive MIMO channel, for the $\mathrm{G} 2+1+1$ symbols are beamformed by using the transmitter side angles, i.e., the $M$ columns of analog beamforming $\mathbf{A}$ are the steering vectors that compose the channel $\mathbf{H}$ shown in Eq. (3) and $\mathbf{D}$ is a diagonal matrix that controls the power of each beam. In such a case, the complete channel state information (CSI) estimation is avoided, so the system saves communication resources by avoiding the use of long pilot sequences [23].

\section{Cancellation of Multi-Layer Interference}

In general, nonlinear detection techniques achieve better performance than linear ones at a cost of increasing the computation burden [13]. We show in this section a nonlinear solution that is a modification of the classical nonlinear SIC algorithm. The method succeeds in mitigating the multi-layer interference without increasing significantly the algorithm complexity compared to the classical SIC algorithm.

The class of SIC detection algorithms are nonlinear decoding methods that detect sequentially the layer data streams. More specifically, the first symbol is obtained at the output of a linear detector that is applied over the received signal. Assuming a perfect symbol decision, this estimate is used to cancel the first layer interference caused in the second layer. After this, another linear filter is applied to extract the second layer symbol. The process is then repeated until the decoder extracts the symbols of all layers [24], [25].

The SIC algorithm works to remove the interference per layer. However, only in the last layer detection phase, the algorithm has collected the total information about interference from the all the other layers, i.e., only the last one has a detection process without interference assuming a perfect estimation in the previous stages. On the other hand, the first layer has poorest detection performance because there is no interference cancellation process in the first stage of the algorithm. The intermediate layers achieve then better performance as more symbols had been detected before. Thus, only last layer enjoys the full diversity.

To deal with the SIC limitation, we use the BRSIC algorithm proposed in [13]. While the first one has a forward recursion loop that cancels out part of inter-layer interference, the second one adds a backward recursion loop to remove the remaining inter-layer interference at the output of the first loop. This modification provides a greater diversity gain than the classical SIC solution, and, consequently, the detection performance of each individual layer increases [20]. A pseudo-code of BRSIC is shown in the Algorithm 1.

Note that, we need to design a filter to extract the layers either for the SIC or BRSIC. Our approach in this paper is to use a minimum mean square error (MMSE) filter $\mathbf{W}=$ $\left[\mathbf{w}_{1}, \ldots, \mathbf{w}_{J}\right]$ to extract the $J$ transmitted layers in the received signal $\mathbf{Y}$ and considering $E_{S}$ the energy of the transmitted signals. More specifically, the algorithm selects the $j$ th column of $\mathbf{W}$, defined as $\mathbf{w}_{j}$, and filters $\mathbf{Y}_{j}$ to extract the $j$ th layer. The output is expressed as $\tilde{\mathbf{x}}_{j}=\mathbf{Y}_{j}^{\mathrm{H}} \mathbf{w}_{j}$.

The design of $\mathbf{W}$ depends on the CSI. However, the channel is compressed due to the beamformed-based transmission scheme that reduces the full massive MIMO channel into an effective one whose expression is given by

$$
\mathbf{H}_{e}=\text { HAD } \in \mathbb{C}^{M \times N_{M S}} .
$$

The use of effective channel information $\mathbf{H}_{e}$ instead of the full massive MIMO channel $\mathbf{H}$ turns out the MMSE filter implementation simpler. This simplicity occurs because the dimension of the matrix $\mathbf{H}_{e}$ is much smaller than the full massive MIMO channel and consequently the matrix inversion in the MMSE filter is less costly. Thus, it is very important to use a precoding solution not only to enable $\mathrm{G} 2+1+1$ scheme, but also to enable less complex detectors solutions in the receiver. In this work, we assume that the effective channel is known at the receiver, and the imperfect channel acquisition case will be considered in a future work due to the lack of space in this letter.

The symbol detection after filtering $\mathbf{w}_{j}$ is represented by the operator $Q(\cdot)$ which means quantization operation in accordance with the employed digital modulation, thereby $\tilde{\mathbf{x}}_{j}$ represents the filtered symbols on the $j$ th layer. The estimated symbol $\hat{\mathbf{x}}_{j}$ is obtained, and its contribution in the other layers is canceled. This yields a modified received signal given per $\mathbf{Y}_{j+1}=\mathbf{Y}_{j}-\mathbf{h}_{e, j} \hat{\mathbf{x}}_{j}^{T}$, where $\mathbf{h}_{e, j}$ is the $j$ th column of the channel matrix $\mathbf{H}_{e}$ and $\mathbf{h}_{e, j} \hat{\mathbf{x}}_{j}^{T}$ represents the estimated interference from the $j$ th layer.

After ending the forward loop, the algorithm starts the backward loop to obtain the improved symbol $\tilde{\mathbf{x}}_{j}^{\prime}$. The process is similar as in the forward loop, in which the modified received signal $\overline{\mathbf{Y}}_{j-1}$ is calculated and filtered by $\mathbf{w}_{j-1}$; the operator $Q\left(\right.$.) is applied over the filter output, defined by $\tilde{\mathbf{x}}_{j-1}^{\prime}$, and returns the estimated symbol $\hat{\mathbf{x}}_{j-1}^{\prime}$.

Assuming that the symbols are estimated perfectly, the SINR of a given layer $j$ of the traditional SIC is given by

$$
\operatorname{SINR}_{j}(j<J)=\frac{E_{s}\left|\mathbf{w}_{j}^{H} \mathbf{h}_{e, j}\right|^{2}}{\sum_{j+1}^{J}\left|\mathbf{w}_{j}^{H} \mathbf{h}_{e, j} E_{s}\right|^{2}+\left\|\mathbf{w}_{j}^{H}\right\|^{2} \sigma_{z}^{2}} .
$$

Considering the BRSIC approach, we will be able to eliminate the first term of the denominator in the backward recursion of the BRSIC, therefore, we will experiment a modified SINR for a given layer $j$ as

$$
\operatorname{SINR}_{j}=\frac{E_{s}\left|\mathbf{w}_{j}^{H} \mathbf{h}_{e, j}\right|^{2}}{\left\|\mathbf{w}_{j}^{H}\right\|^{2} \sigma_{z}^{2}} .
$$

Then, we can argue the improvement at the BER performance due to the addition of the backward recursion of the BRSIC.

Assuming the channel is known at MS, the detection of each symbol layer is enhanced by the beam high gains which are achieved due to the massive MIMO array. Therefore, the use of $\mathrm{HB}$ increases the correct decision rate of the BRSIC receiver, i.e., the bit error rate (BER) decreases. A more detailed description of such an algorithm is found in [13] and [26]. 
Note that the analog beamforming selects the set of directions to be used in the transmission, and, consequently the channel dimensions are reduced to the order of number of RF chains. The equivalent channel is far smaller than the full massive MIMO channel because the number of RF chains is smaller than the number of antennas. Therefore, one advantage of using $\mathrm{HB}$ is that the BRSIC computational burden increases with the number of RF chains instead of the number of antennas. The computational complexity of the proposed algorithm is calculated in function of the MMSE filter computation and the operations performed in the forward and backward loops. The dominant complexity cost is associated with the inverses of the matrices. In accordance with [27], the computational complexity of the MMSE filter is obtained based on the inverse of a square matrix of size $N_{M S} \times N_{M S}$, which is $\mathcal{O}\left(N_{M S}^{3}\right)$, and the complexity of the matrix product between a square matrix of size $N_{M S} \times N_{M S}$ and a rectangular one $N_{M S} \times M$, which is $O\left(M N_{M S}^{2}\right)$. Moreover, the number of operations inside each loop is approximately $O\left(N_{M S}\right)$. Therefore, the overall complexity is approximately $\mathcal{O}\left(\max \left(N_{M S}^{3}, M N_{M S}^{2}, N_{M S}\right)\right)$. Thus, we observe that the complexity of the BRSIC algorithm is approximately $O\left(N_{M S}^{3}\right)$ and it corresponds to the same computational cost of the SIC algorithm.

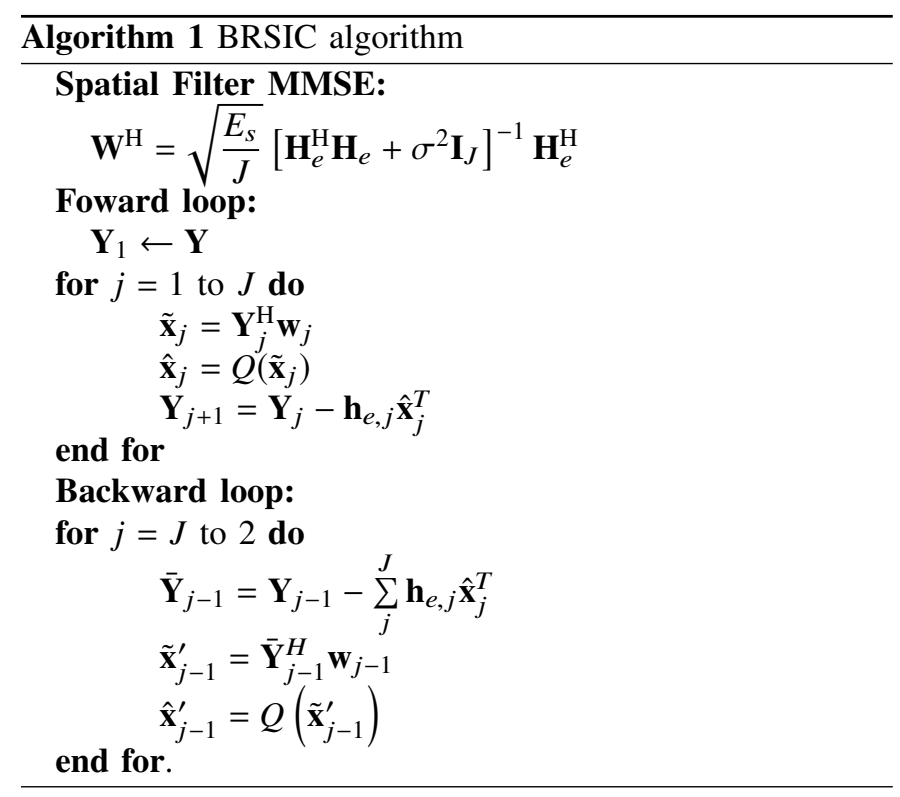

\section{Simulation Results}

In this section, we evaluate our hybrid beamforming solution, which combines the enhanced spatial multiplexing capabilities of the large array and the robustness of $\mathrm{G} 2+1+1$ scheme. At the BS, the beamforming is implemented with $N_{B S}=128$ transmitter antennas and $M=4 \mathrm{RF}$ chains. The channel is generated using Eq. (3), where the angles are defined for two different scenarios. One has a set of angles that configures a high angular spread scenario while the second set defines a low angular spread one. At the receiver, we separate the data streams of each transmission layer by designing nonlinear and linear decoders assuming the number of antennas $N_{M S}=4$. To compare the proposed transmission scheme with different receiver solutions, we evaluated them in terms of BER assuming a QPSK modulation.

Fig. 2 shows the system performance considering a high angular spread scenario. The angles of departure and arrival in such a case are $\theta_{T X, l}$ and $\theta_{R X, l}$, with a angle spacing which varies of $15^{\circ}$ to $90^{\circ}$. Using the proposed hybrid beamforming scheme, the BS encodes $K=4$ data streams using the $\mathrm{G} 2+1+1$ scheme, and the receiver employs a BRSIC nonlinear decoder to separate them. This solution is compared with the case where there is no $\mathrm{G} 2+1+1$ encoder at the transmitter and the receiver employs either a simple linear decoder, which can be zero forcing $(\mathrm{ZF})$ and MMSE, or a nonlinear decoder [26], which is a the BRSIC solution.

The HB-ZF and HB-MMSE curves show the BER performance using a $\mathrm{ZF}$ and MMSE receivers, respectively. The curves converge to the same performance as the SNR increases. However, the results show the ZF and MMSE limitations, where their BER performance achieves only $10^{-3}$ even at very high SNR. One way to improve the BER consist of using a nonlinear receiver as can be noticed by the curve HB-BRSIC. This is because the symbol detection per layer is better than that performed by the linear decoders. Using the symbols detected per layer, the algorithm cancels out the interference in every layer. Therefore, the BRSIC solves locally multiple MMSE problems always taking into account the decision from the last layer. In the low SNR regime, the probability of taking the wrong symbol decision per layer is high resulting in a small gain with respect to the other linear receivers. The use of a $\mathrm{G} 2+1+1$ combined with the $\mathrm{HB}$ gain provides great performance in both high and low SNRs. The robustness inserted by the Alamouti layer reduces the probability of wrong decisions on the BRSIC steps and consequently improves BER system performance.

The Fig. 3 shows the BER performance for a low angular spread scenario. In such a case, the angles of departure and arrival, $\theta_{T X, l}$ and $\theta_{R X, l}$, have a angle spacing of $\pi / 20$. Assuming a BS equipped with $N_{B S}=M=4$ transmitter antennas and a receiver with $N_{M S}=4$ receive antennas, the HB-BRSIC curve shows a poor performance due to the low angular spread of the channel that generates a strong interference among the layers. The insertion of the $\mathrm{G} 2+1+1$ scheme provides a better performance to the system, however the benefits are only enjoyed under high SNR conditions. Note that the interference among the layers is the limiting factor that cannot be efficiently handled with few antennas. Using a massive array, the BS has a high spatial definition that can be used to mitigate such a interference by properly designing the beamforming. The proposed solution combines such a spatial selectness with the $\mathrm{G} 2+1+1$ encoder robustness to reduce the interference among the layers. This explains the result expressed by the curve that represents a 128x4 MIMO system.

\section{CONCLUSION}

In this work, we propose a multi-layer beam transmission scheme for massive MIMO that exploits, simultaneously, high 


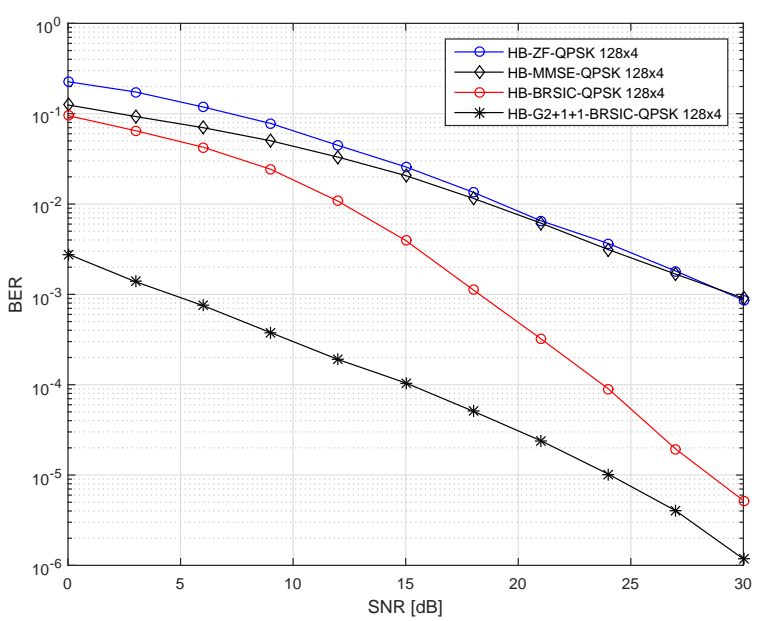

Figure 2. BER performance for a BS employing $\mathrm{HB}$ with $\mathrm{G} 2+1+1$ and without $\mathrm{G} 2+1+1$. The plots are obtained for different decoder solutions:ZF, MMSE and BRSIC.

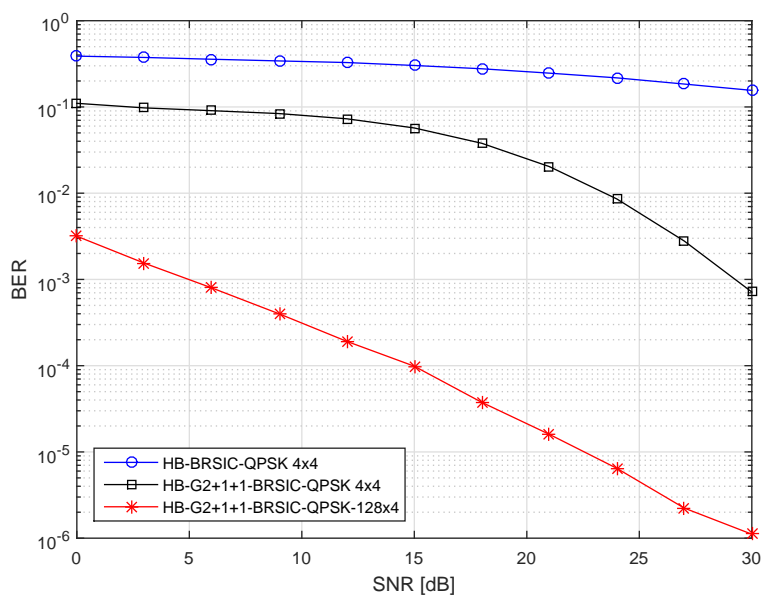

Figure 3. BER for a BS employing the proposed HB scheme for low angular spread scenarios.

beamforming, spatial multiplexing and diversity gains. The transmission is done by means of hybrid beamforming solution, thus the gain obtained by the large array can be used to point the signal at a specific direction. The encoder used follows the $\mathrm{G} 2+1+1$ configuration that provides, at the same time, spatial multiplexing and diversity gains. Furthermore, the receiver employs a BRSIC nonlinear decoder.

The proposed scheme provides great robustness in scenarios with either low or high SNR. This is because our solution mainly combines the high gain of massive MIMO and the robustness of the G2+1+1 scheme. Furthermore, we also achieved good performances in low angular spread conditions, where the poor channel in spreaders increases the interference among the data streams. The results showed that the proposed scheme is an attractive solution to be used by massive MIMO systems to enhance their BER performance even in challenging situations, as for instance in low SNR and/or low angular spread scenarios. Another interesting aspect is that the analog beamforming is constructed only based on the angular information of the channel which means that the BS does not need to acquire the whole the channel matrix to design the proposed HB solution. As a continuation of this work, we will extend our solution to cope with the multi-user scenario.

\section{REFERENCES}

[1] R. C. de Lamare, "Massive MIMO systems: Signal processing challenges and future trends", URSI Radio Science Bulletin, vol. 86, no. 4, pp. 8-20, Dec. 2013, ISSN: 1024-4530. DOI: 10.23919/URSIRSB.2013.7909827.

[2] A. Alkhateeb, O. E. Ayach, G. Leus, and R. W. Heath, "Channel estimation and hybrid precoding for millimeter wave cellular systems", IEEE Journal of Selected Topics in Signal Processing, vol. 8, no. 5, pp. 831-846, Oct. 2014, ISSN: 19324553. DOI: 10.1109/JSTSP.2014.2334278.

[3] E. G. Larsson, O. Edfors, F. Tufvesson, and T. L. Marzetta, "Massive MIMO for next generation wireless systems", IEEE Communications Magazine, vol. 52, no. 2, pp. 186-195, Feb. 2014, ISSN: 0163-6804. DOI: 10.1109/MCOM.2014.6736761.

[4] J. Choi, H. Yu, and Y. H. Lee, "Adaptive MIMO decision feedback equalization for receivers with time-varying channels", IEEE Transactions on Signal Processing, vol. 53, no. 11, pp. 4295-4303, Nov. 2005, ISSN: 1053-587X. DOI: 10.1109/ TSP.2005.857053.

[5] A. A. Rontogiannis, V. Kekatos, and K. Berberidis, "A squareroot adaptive V-BLAST algorithm for fast time-varying MIMO channels", IEEE Signal Processing Letters, vol. 13, no. 5, pp. 265-268, May 2006, ISSN: 1070-9908. DOI: 10.1109/LSP. 2006.870373

[6] W. d. C. Freitas Jr, F. R. Cavalcanti, and R. R. Lopes, "Hybrid transceiver schemes for spatial multiplexing and diversity in MIMO systems", Journal of Communication and Information systems (JCIS), vol. 20, no. 3, pp. 63-76, 2005. DOI: 10.14209/ jcis.2005.21.

[7] F. Sohrabi and W. Yu, "Hybrid digital and analog beamforming design for large-scale MIMO systems", in IEEE International Conference on Acoustics, Speech and Signal Processing (ICASSP), Apr. 2015, pp. 2929-2933. DOI: 10.1109/ICASSP. 2015.7178507.

[8] C. Kim, T. Kim, and J. Y. Seol, "Multi-beam transmission diversity with hybrid beamforming for MIMO-OFDM systems", in IEEE Globecom Workshops (GC WKSHPS), Dec. 2013, pp. 61-65. DOI: 10.1109/GLOCOMW.2013.6824962.

[9] S. Sun, T. S. Rappaport, R. W. Heath, A. Nix, and S. Rangan, "MIMO for millimeter-wave wireless communications: Beamforming, spatial multiplexing, or both?", IEEE Communications Magazine, vol. 52, no. 12, pp. 110-121, Dec. 2014, ISSN: 0163-6804. DOI: 10.1109/MCOM.2014.6979962.

[10] X. Meng, X. G. Xia, and X. Gao, "Omnidirectional stbc design in massive MIMO systems", in IEEE Global Communications Conference (GLOBECOM), Dec. 2015, pp. 1-6. DOI: 10.1109/ GLOCOM.2015.7417291.

[11] _ " "Omnidirectional space-time block coding for common information broadcasting in massive MIMO systems", IEEE Transactions on Wireless Communications, vol. PP, no. 99, pp. 1-1, 2017, ISSN: 1536-1276. DOI: 10.1109/TWC. 2016. 2622259

[12] X. G. Xia and X. Gao, "A space-time code design for omnidirectional transmission in massive MIMO systems", IEEE Wireless Communications Letters, vol. 5, no. 5, pp. 512-515, Oct. 2016, ISSN: 2162-2337. DOI: 10 . 1109/LWC . 2016. 2597231

[13] W. C. Freitas Jr., D. C. Moreira, C. C. Cavalcante, and A. L. F. de Almeida, "Backward recursion in layered spacetime non-linear interference cancellation detectors", Journal of Communication and Information Systems (JCIS), vol. 26, pp. 30-34, Apr. 2011. DOI: 10.14209/jcis.2011.4. 
[14] J. Benesty, Y. Huang, and J. Chen, "A fast recursive algorithm for optimum sequential signal detection in a BLAST system", IEEE Transactions on Signal Processing, vol. 51, no. 7, pp. 1722-1730, Jul. 2003, ISSN: 1053-587X. DOI: 10.1109/ TSP.2003.812897.

[15] G. J. Foschini, G. D. Golden, R. A. Valenzuela, and P. W. Wolniansky, "Simplified processing for high spectral efficiency wireless communication employing multi-element arrays", IEEE Journal on Selected Areas in Communications, vol. 17, no. 11, pp. 1841-1852, Nov. 1999, ISSN: 0733-8716. DOI: $10.1109 / 49.806815$.

[16] Y. Shang and X. G. Xia, "An improved fast recursive algorithm for V-BLAST with optimal ordered detections", in 2008 IEEE International Conference on Communications, May 2008, pp. 756-760. DOI: 10.1109/ICC.2008.148.

[17] A. Rozé, M. Crussière, M. Hélard, and C. Langlais, "Comparison between a hybrid digital and analog beamforming system and a fully digital massive MIMO system with adaptive beamsteering receivers in millimeter-wave transmissions", in International Symposium on Wireless Communication Systems (ISWCS), Sep. 2016, pp. 86-91. DOI: 10.1109/ISWCS.2016. 7600880.

[18] D. C. Araújo, E. Karipidis, A. L. F. de Almeida, and J. C. M. Mota, "Improving spectral efficiency in large-array FDD systems with hybrid beamforming", in IEEE Sensor Array and Multichannel Signal Processing Workshop (SAM), Jul. 2016, pp. 1-5. DOI: 10.1109/SAM.2016.7569692.

[19] P. Xia, S. K. Yong, J. Oh, and C. Ngo, "Multi-stage iterative antenna training for millimeter wave communications", in IEEE Global Telecommunications Conference (GLOBECOM), Nov. 2008, pp. 1-6. DOI: 10.1109/GLOCOM.2008.ECP.908.

[20] F. M. C. Caldas, D. C. Araújo, and W. C. Freitas, "Hybrid MIMO schemes with MRT precoding and modified SIC receiver", in 2014 IEEE Latin-America Conference on Communications (LATINCOM), Nov. 2014, pp. 1-5. DOI: 10.1109/ LATINCOM.2014.7041854.

[21] T. S. Rappaport, F. Gutierrez, E. Ben-Dor, J. N. Murdock, Y. Qiao, and J. I. Tamir, "Broadband millimeter-wave propagation measurements and models using adaptive-beam antennas for outdoor urban cellular communications", IEEE Transactions on Antennas and Propagation, vol. 61, no. 4, pp. 1850-1859, Apr. 2013, ISSN: 0018-926X. DOI: 10.1109/ TAP.2012.2235056.

[22] T. Obara, S. Suyama, J. Shen, and Y. Okumura, "Joint fixed beamforming and eigenmode precoding for super high bit rate massive MIMO systems using higher frequency bands", in 2014 IEEE 25th Annual International Symposium on Personal, Indoor, and Mobile Radio Communication (PIMRC), Sep. 2014, pp. 607-611. DOI: 10.1109/PIMRC.2014.7136237.

[23] S. Noh, M. D. Zoltowski, and D. J. Love, "Training sequence design for feedback assisted hybrid beamforming in massive MIMO systems", IEEE Transactions on Communications, vol. 64 , no. 1, pp. 187-200, Jan. 2016, ISSN: 0090-6778. DOI: 10.1109/TCOMM.2015.2498184.

[24] R. Fa and R. C. D. Lamare, "Multi-branch successive interference cancellation for MIMO spatial multiplexing systems: Design, analysis and adaptive implementation", IET Communications, vol. 5, no. 4, pp. 484-494, Mar. 2011, ISSN: 17518628. DOI: 10.1049 /iet-com.2009.0843.

[25] P. Li, R. C. de Lamare, and R. Fa, "Multiple feedback successive interference cancellation detection for multiuser MIMO systems", IEEE Transactions on Wireless Communications, vol. 10, no. 8, pp. 2434-2439, Aug. 2011, ISSN: 1536-1276. DOI: 10.1109/TWC.2011.060811.101962.

[26] Y. Cho, J. Kim, W. Yang, and C. Kang, MIMO-OFDM Wireless Communications with MATLAB, 1st ed. Wiley-IEEE Press, 2010, pp. 419-429, ISBN: 9780470825631. DOI: $10.1002 /$ 9780470825631.fmatter.

[27] G. H. Golub and C. F. Van Loan, Matrix computations. JHU Press, 2012, vol. 3.

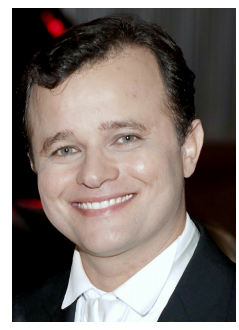

Francisco M. C. Caldas. Francisco Márcio C Caldas received Bachelor degree in Telematics from Federal Institute of Education, Science and Technology of Ceará (IFCE) and his M.S. degree in teleinformatics from the Federal University of Ceará (UFC) in 2010 and 2012, respectively. He is currently pursuing his $\mathrm{PhD}$ degree at Federal University of Ceará (UFC). He is member of Wireless Telecommunications Research Group (GTEL), Fortaleza, Brazil. His research interests are Massive MIMO, Millimeter Wave, Hybrid Beamforming and

nonlinear detection.

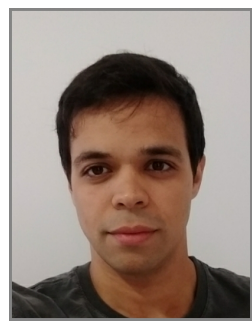

Daniel C. Araujo. Daniel Costa Araújo - received his Bachelor degree in Telecommunications Engineering from Fortaleza University (UNIFOR) in 2010 and his M.S. and PhD degrees in Teleinformatics Engineering from Federal University of Ceará (UFC), Brazil in 2012 and 2016, respectively. During his studies, he was supported by the Brazilian agency CAPES and Ericsson. During his PhD, he was visiting researcher at ERICSSON in 2013 and 2015 up to 2016 . He is currently a post-doc researcher of Wireless Telecommunication Research Group (GTEL). His areas of interests concern to channel estimation, sparse signal processing, massive MIMO and Hybrid beamforming.

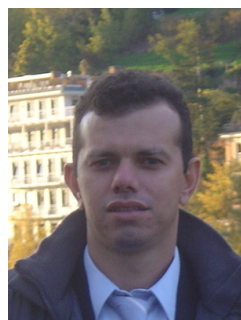

Walter C. Freitas Jr. Walter C. Freitas Jr. - received his $\mathrm{PhD}$ degree in Teleinformatic Engineering from Federal University of Ceará (UFC), Brazil in 2006 and his B.S. and M.S. degrees in Electrical Engineering from the same university. During his studies, he was supported by the Brazilian agency FUNCAP and Ericsson. During Q3 of 2015 up to Q2 of 2016, he was a Post-doc Researcher at I3S/CNRS Laboratory, from the University of Nice, Sophia Antipolis, France. During 2005 Walter Freitas Jr. was a senior research of Nokia Technology Institute, He is currently an Assistant Professor with the Department of Teleinformatics Engineering of the Federal University of Ceará and researcher of Wireless Telecom Research Group (GTEL) one of the most important research groups in telecommunication in Brazil. His main area of interest concerns features development to improve the performance of the wireless communication systems, multilinear algebra, and tensor-based signal processing applied to communications and interference avoidance tools. 